\title{
Clinical characteristics of a group of patients with multiple myeloma who had two different $\lambda$ light chains by immunofixation electrophoresis: A retrospective study from a single center
}

\author{
GUANGZHONG YANG, CHUANYING GENG and WENMING CHEN
}

\author{
Department of Hematology and Multiple Myeloma Research Center of Beijing, Beijing Chaoyang Hospital, \\ Capital Medical University, Beijing 100020, P.R. China
}

Received April 7, 2014; Accepted February 13, 2015

DOI: $10.3892 / \mathrm{etm} .2015 .2352$

\begin{abstract}
The objective of the present study was to conduct a retrospective analysis of the clinical characteristics of a group of patients with multiple myeloma (MM) who had two different immunoglobulin $\lambda$ light chains as determined by immunofixation electrophoresis (IFE). A total of 26 patients with MM had two different $\lambda$ light chains by IFE at diagnosis in hospital from January 2006 to June 2012. Data were collected from the medical records of these patients. The clinical characteristics, laboratory parameters, responses, risk factors and outcomes of this special group of patients were retrospectively analyzed. The patients with MM and two different $\lambda$ light chains by IFE accounted for 5.4\% (26/483) of all the patients with MM during the same period. There were 17 patients $(65.4 \%)$ with renal dysfunction and 10 patients $(38.5 \%)$ with extramedullary plasmacytoma (EMP) at diagnosis. Ten patients $(38.5 \%)$ had abnormalities by fluorescence in situ hybridization (FISH) including $\mathrm{t}(4 ; 14), \mathrm{t}(14 ; 16)$ and/or del17p. Of these patients, three (11.5\%) achieved complete remission (CR), six (23.1\%) very good partial remission (VGPR), six (23.1\%) partial remission (PR) and the overall remission (OR) rate was $57.7 \%(15 / 26)$. The OR rate of the patients who received chemotherapy with bortezomib was superior to that of the patients who received chemotherapy without bortezomib $(78.6 \%$ vs. $33.3 \%$; $\mathrm{P}<0.05)$. The OR rate of the patients who received autologous stem cell transplantation (SCT) was superior to that of the patients who did not receive autologous SCT $(77.8 \%$ vs. $47.1 \%$; $\mathrm{P}<0.05)$. The median overall survival (OS) was 12.6 months (range, 2-38 months) in this study. The patients who received chemotherapy with bortezomib had a significantly longer median OS as compared with the patients who received chemotherapy
\end{abstract}

Correspondence to: Professor Wenming Chen, Department of Hematology and Multiple Myeloma Research Center of Beijing, Beijing Chaoyang Hospital, Capital Medical University, 8 Gongrentiyuchangnanlu, Beijing 100020, P.R. China

E-mail: myeloma@163.com

Key words: multiple myeloma, $\lambda$ light chain, prognosis without bortezomib (19 vs. 6 months; $\mathrm{P}=0.049$ ). The patients who received autologous SCT also had significantly longer median OS as compared with the patients who did not receive autologous SCT (18 vs. 7 months; $\mathrm{P}=0.041)$. The median duration of remission (DOR) was 5 months (range, 0-28 months). The patients who received bortezomib or autologous SCT had significantly longer median DOR than those who did not ( $\mathrm{P}=0.046$ and $\mathrm{P}=0.012$, respectively). The patients with abnormalities by FISH had similar OR rates, median OS and median DOR as compared with the patients without abnormalities by FISH and there was no statistically significant difference in OR rate, median OS and median DOR between the patients with EMP and those without. The MM patients with two different $\lambda$ light chains by IFE may have specific characteristics, and are accompanied by a high incidence of renal dysfunction and EMP. They have a poor prognosis and require novel therapy including bortezomib and autologous SCT.

\section{Introduction}

Multiple myeloma (MM) is caused by the uncontrolled proliferation of monoclonal plasma cells, resulting in the production of monoclonal immunoglobulin ( $\mathrm{Ig}$ ) and substantial immunosuppression and endorgan damage, including direct and indirect effects on the blood, skeleton, and kidneys (1).

Recent treatment options for MM have led to improved response rates and increased survival. However, MM is a heterogeneous disease with variable disease course and response to therapy, and a survival outcome that ranges from less than 1 year to more than 10 years (1). Various clinical characteristics, including age and type of M-component, have been identified to predict the disease course as well as outcome in patients with myeloma. In the past ten years, many factors have been found to impact the prognosis, including stage according to the International Staging System (ISS) (2), renal function (3), cytogenetics (4) and extramedullary plasmacytoma (EMP) (5). It is extremely important for treatment strategies to include the evaluation of prognostic factors and risk stratification (6).

The present study relates to a special series of patients with MM. These patients were found to have two different Ig $\lambda$ light chains by immunofixation electrophoresis (IFE) at diagnosis, and they had certain similarities in their clinical 
characteristics. However, to the best of our knowledge, there have been no previous reports associated with such patients. Therefore, the characteristics of these patients were summarized retrospectively and their prognosis was analyzed.

\section{Patients and methods}

Patients. A total of 483 patients were diagnosed with MM in Beijing Chaoyang Hospital (Beijing, China) from January 2006 to June 2012. Among them, 26 patients (5.4\%) had two different $\lambda$ light chains when analyzed by IFE. Data were collected from the medical records of such patients. All the patients received baseline evaluations prior to treatment which included physical examination, blood counts, hepatic and renal function tests, bone marrow aspirate and biopsy, serum and urine protein electrophoreses, and quantitation of serum Igs and urinary light chains, serum $\beta 2$ microglobulin and C-reactive protein. A chest X-ray and a complete radiological bone survey were also performed. Detection by fluorescence in situ hybridization (FISH) of $\mathrm{t}(4 ; 14), \mathrm{t}(14 ; 16)$ and del17p was completed prior to treatment.

Treatment strategies. The patients received various treatment strategies. Some were treated with combination regimens including the $\mathrm{PD} \pm \mathrm{T}$ regimen (bortezomib and dexamethasone with or without thalidomide) or the PAD regimen (bortezomib, doxorubicin and dexamethasone). Other patients were treated with conventional regimens without bortezomib including the VAD regimen (vindesine, doxorubicin and dexamethasone), the TAD regimen (thalidomide, doxorubicin and dexamethasone) or the $\mathrm{TD} \pm \mathrm{CTX}$ regimen (thalidomide, dexamethasone with or without cyclophosphamide). The patients who were suitable for autologous stem cell transplantation (SCT) received autologous SCT. Local radiotherapy was used for the patients with extramedullary plasmacytoma (EMP) following total therapy.

In addition to antimyeloma treatment, all patients received intensive supportive care including intravenous hydration, alkalinization of urine, correction of hypercalcemia and the discontinuation of all potential nephrotoxic agents. Renal dialysis was offered to patients with severe renal dysfunction.

Statistical analysis. All statistical analyses were performed with SPSS 13.0 statistical software (SPSS, Inc., Chicago, IL, USA). The efficacy was evaluated by Chi-square test. Survival analysis was performed with a life-table and Kaplan-Meier survival curve. A P-value of $<0.05$ was considered significant for all statistical calculations. Response was evaluated following the international uniform response criteria for multiple myeloma defined by the International Myeloma Working Group (7). The main indices were overall remission (OR), complete remission (CR), very good partial remission (VGPR) and partial remission (PR) rates, in which OR is equal to the sum of CR, VGPR and PR. Overall survival (OS) and duration of remission (DOR) were used to establish the durability of response in this study. The OS is the duration from diagnosis of the disease to mortality (regardless of cause of mortality) or loss to follow-up. The DOR is the duration from the first observation of PR to the time of disease progression or mortality from a cause other than disease progression (regardless of the nature of this cause), whichever occurred first.
Table I. Characteristics of the patients with multiple myeloma at diagnosis.

\begin{tabular}{lc}
\hline Characteristics & Values \\
\hline $\begin{array}{l}\text { Number of patients } \\
\text { Gender, } \mathrm{n}(\%)\end{array}$ & 26 \\
Male & $23(88.5)$ \\
Female & $3(11.5)$ \\
Median age, years (range) & $59(48-76)$ \\
Type of M-component, $\mathrm{n}(\%)$ & \\
IgG- $\lambda, \lambda$ & $14(53.8)$ \\
IgA- $\lambda, \lambda$ & $7(26.9)$ \\
IgD- $\lambda, \lambda$ & $5(19.2)$ \\
Durie-Salmen stage, $\mathrm{n}(\%)$ & \\
IIa & $2(7.7)$ \\
IIIa & $7(26.9)$ \\
IIIb & $17(65.4)$
\end{tabular}

ISS stage, $\mathrm{n}(\%)$

I

III

$\operatorname{WBC}\left(\times 10^{9} / 1\right)$, median (range)

$5.6(2.2-7.9)$

$\mathrm{Hb}(\mathrm{g} / \mathrm{l})$, median (range)

$68(52-119)$

$\operatorname{PLT}\left(\times 10^{9} / 1\right)$, median (range)

$89(43-201)$

Serum album (g/l), median (range)

$24(17-38)$

Serum creatinine $(\mu \mathrm{mol} / \mathrm{l})$, median (range)

$342(67-1455)$

Plasma cell (BM, \%), median (range)

$41.5(11.0-85.0)$

Extramedullary plasmacytoma, $\mathrm{n}(\%)$

Hypercalcemia n (\%)

Abnormalities by FISH, n (\%)

del17p

$\mathrm{t}(14 ; 16)$

$3(11.5)$

$5(19.2)$

$\mathrm{t}(4 ; 14)$

$16(61.5)$

None of the above

$6.0(3.1-18.6)$

Serum $\beta 2$ microglobulin ( $\mathrm{mg} / \mathrm{l})$, median (range)

ISS, International Staging System; WBC, white blood cell; $\mathrm{Hb}$, hemoglobin; PLT, platelet; BM, bone marrow; FISH, fluorescence in situ hybridization.

\section{Results}

Patient characteristics. There were 23 males and 3 females with a median age of 59 years (range, 48-76 years) in this study (Table I). All the patients were found to have two different $\lambda$ light chains following IFE tests (Fig. 1). Among these patients, $\operatorname{IgG} \mathrm{MM}$ was found in 14 patients (53.8\%), $\operatorname{IgA}$ in 7 patients (26.9\%) and $\mathrm{IgD}$ in 5 patients $(19.2 \%)$. Seventeen patients $(65.4 \%)$ had renal dysfunction, 12 patients $(46.2 \%)$ in the group received hemodialysis and 10 patients (38.5\%) had extramedullary plasmacytoma (EMP) at diagnosis. 
Table II. Efficacy of the different treatment strategies.

\begin{tabular}{lcccrr}
\hline Regimen & No. & CR (n) & VGPR (n) & PR (n) & OR rate (\%) \\
\hline Bortezomib without auto-SCT & 9 & 1 & 3 & 2 & 1 \\
Bortezomib with auto-SCT & 5 & 2 & 2 & 06.7 \\
No bortezomib without auto-SCT & 8 & 0 & 1 & 2 & 1 \\
No bortezomib with auto-SCT & 4 & 0 & 6 & 6 & 50.0 \\
Total & 26 & 3 & 57.7 \\
\hline
\end{tabular}

Bortezomib, regimen combination with bortezomib; auto-SCT, autologous stem cell transplantation; no bortezomib, regimen combination without bortezomib; CR, complete remission; VGPR, very good partial remission; PR, partial remission; OR, overall remission.

A

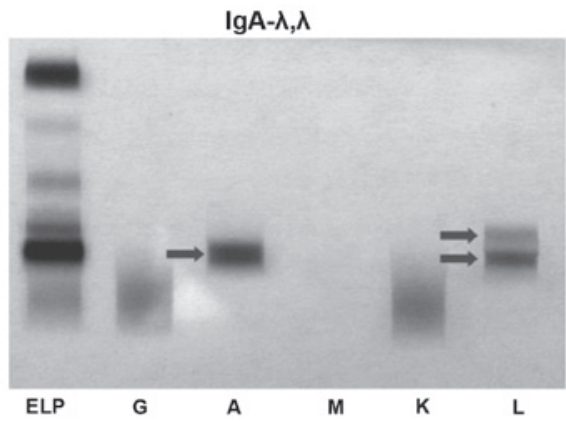

B

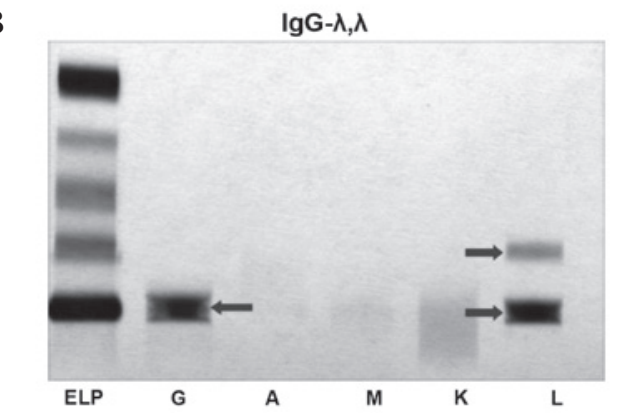

C

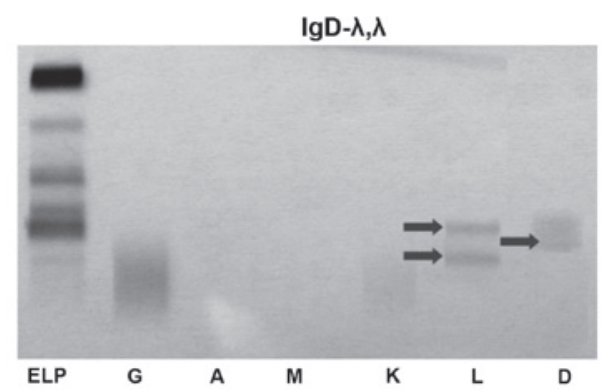

Figure 1 . Two bands for the immunoglobulin (Ig) $\lambda$ light chain by immunofixation electrophoresis (IFE). (A) $\operatorname{Ig} A-\lambda, \lambda$, (B) $\operatorname{IgG}-\lambda, \lambda$ and (C) $\operatorname{IgD}-\lambda, \lambda$. Lane ELP, serum protein electrophoresis; lane G, IgG; lane A, IgA; lane M, $\mathrm{IgM}$; lane $\mathrm{K}, \kappa$ light chain; lane $\mathrm{L}, \lambda$ light chain; lane $\mathrm{D}, \operatorname{IgD}$.

Twenty-two patients (84.6\%) had stage III disease according to the ISS. Five patients had hypercalcemia at diagnosis. Ten patients $(38.5 \%)$ had $\mathrm{t}(4 ; 14), \mathrm{t}(14 ; 16)$ or del17p abnormalities by FISH analysis. Other characteristics are presented in Table I.

Response analysis. Three patients (11.5\%) achieved CR, 6 patients $(23.1 \%)$ VGPR, 6 patients $(23.1 \%)$ PR and the OR rate was $57.7 \%(15 / 26)$. Fourteen patients received chemotherapy with bortezomib and five of them continued to receive autolo-
Table III. DOR of the patients affected by different factors.

\begin{tabular}{lccc}
\hline Factor & $\begin{array}{c}\text { Number } \\
\text { of patients }\end{array}$ & $\begin{array}{c}\text { Median } \\
\text { DOR (months) }\end{array}$ & P-value \\
\hline $\begin{array}{l}\text { Bortezomib } \\
\text { Yes }\end{array}$ & 14 & 11 & 0.046 \\
No & 12 & 4 & \\
Auto-SCT & & & \\
Yes & 9 & 16 & 0.012 \\
No & 17 & 4.5 & \\
Abnormality by FISH & & & \\
Yes & 10 & 6 & 0.617 \\
No & 16 & 9 & \\
EMP & & & \\
Yes & 10 & 6.5 & 0.667 \\
No & 16 & 9 & \\
\hline
\end{tabular}

DOR, duration of remission; auto-SCT, autologous stem cell transplantation; abnormality by FISH, one of $\mathrm{t}(4 ; 14), \mathrm{t}(14 ; 16)$ or del17p abnormalities by FISH; FISH, fluorescence in situ hybridization; EMP, extramedullary plasmacytoma.

gous SCT. Twelve patients received chemotherapy without bortezomib and four of them proceeded to receive autologous SCT. The results are presented in Table II.

The OR rate of the 14 patients who received chemotherapy with bortezomib was superior to that of the 12 patients who received chemotherapy without bortezomib (78.6\% vs. 33.3\%, respectively; $\mathrm{P}<0.05)$. The $\mathrm{OR}$ rate of the 9 patients who received autologous SCT was superior to that of the 17 patients who did not receive autologous SCT (77.8\% vs. $47.1 \%$, respectively; $\mathrm{P}<0.05)$.

Ten patients $(10 / 26,38.5 \%)$ had $t(4 ; 14), t(14 ; 16)$ or del17p cytogenetic abnormalities by FISH in this study. Seven of them received chemotherapy with bortezomib and two of them received autologous SCT. Their OR rate was $60.0 \%$ and the OR rate of the patients without abnormality by FISH was $56.3 \%(\mathrm{P}>0.05)$.

Among the patients with EMP $(n=10)$, five received chemotherapy with bortezomib and one received autologous SCT. 
$\mathbf{A}$

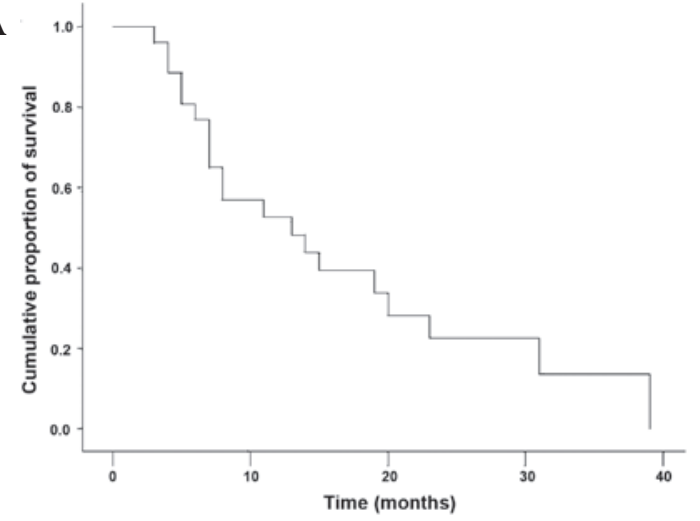

C

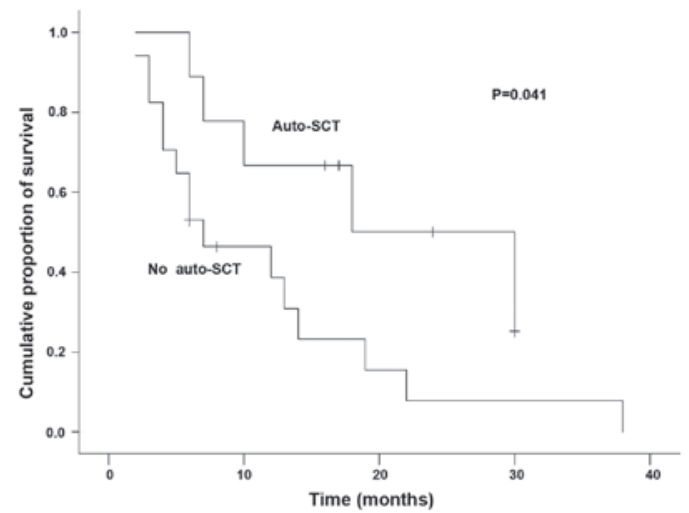

$\mathbf{E}$

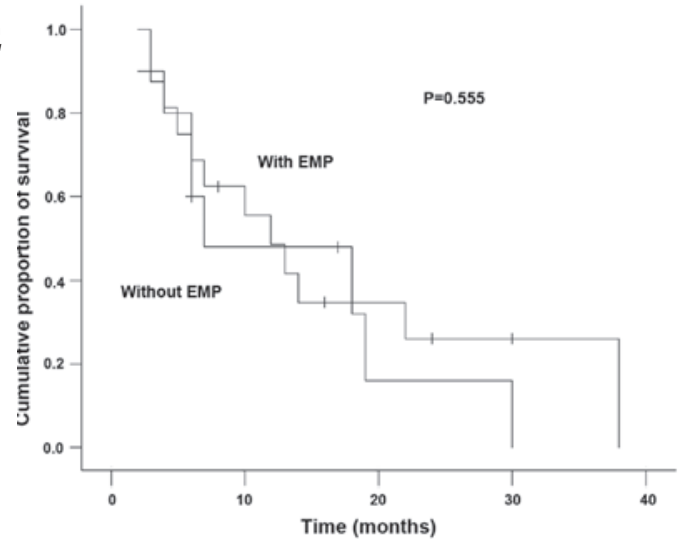

B

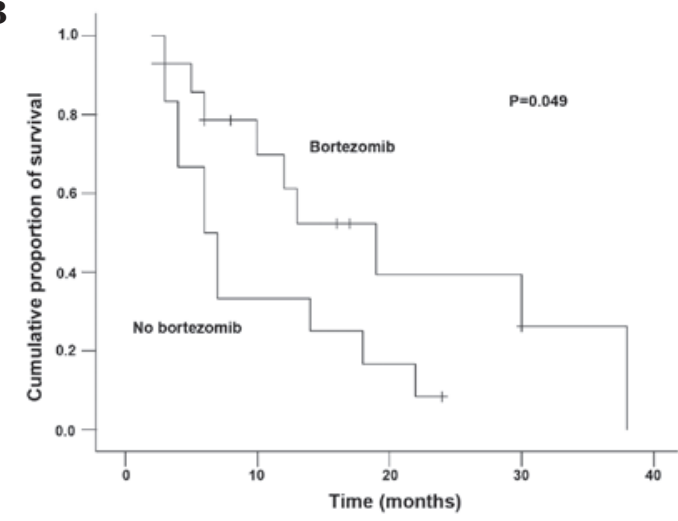

D

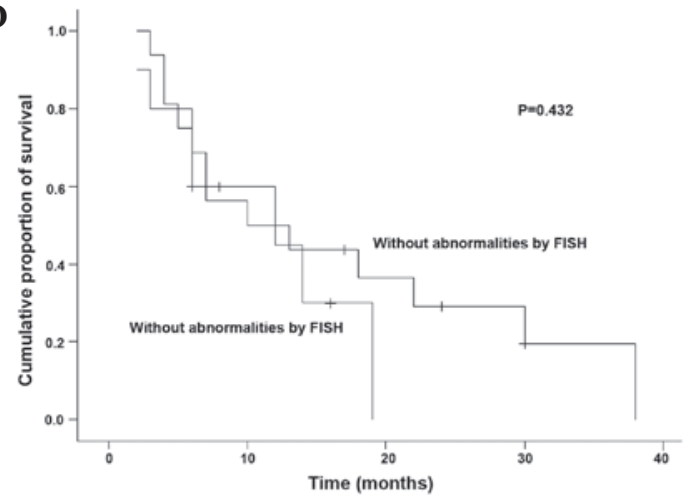

$\mathbf{F}$

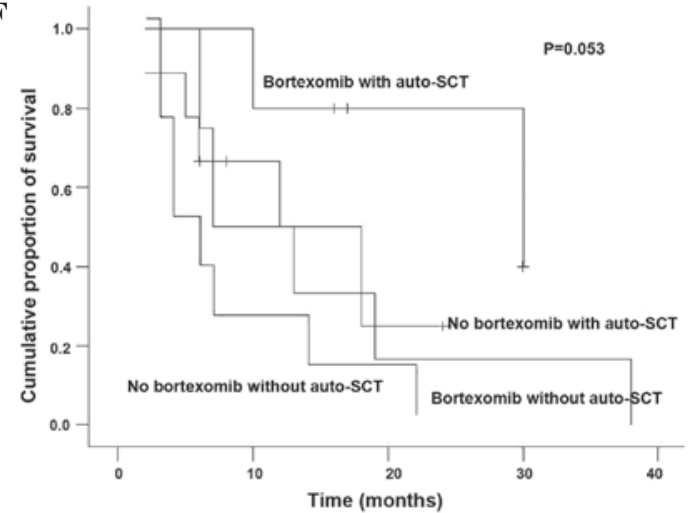

Figure 2. (A) Overall survival of patients with two bands for the $\lambda$ light chain when analyzed by IFE ( $\mathrm{n}=26$ ) and overall survival according to the factors (B and F) bortezomib, (C and F) autologous SCT, (D) abnormalities by FISH and (E) EMP. IFE, immunofixation electrophoresis; SCT, stem cell transplantation; FISH, fluorescence in situ hybridization; EMP, extramedullary plasmacytoma.

One of these patients achieved CR, two achieved VGPR and two achieved PR. There was no statistically significant difference on the OR rate between the patients with EMP and the patients without EMP (50.0\% vs. $62.5 \%$, respectively; $\mathrm{P}>0.05)$.

Prognosis. The endpoint of follow-up was patient mortality, loss to follow-up or October 31,2012. No case in the study was lost during the period of follow-up. At the time of analysis, $6 / 26$ patients $(23.1 \%)$ were alive, with a median follow-up of 24 months. The median OS was 12.6 months (range, 2-38 months; Fig. 2A). The patients who received chemotherapy with bortezomib had a significantly longer median OS as compared with the patients who received chemotherapy without bortezomib (19 vs. 6 months, respectively; $\mathrm{P}=0.049$; Fig. 2B). The patients who received autologous SCT $(n=9)$ also had a significantly longer median OS as compared with the patients who did not receive autologous SCT (18 vs. 7 months, respectively; $\mathrm{P}=0.041$; Fig. 2C). However, the patients with abnormalities by FISH had similar median OS as compared with the patients without abnormalities by FISH (18 vs. 10 months, respectively; $\mathrm{P}=0.432$; Fig. 2D). There was no statistically significant difference on median OS between the patients with EMP and those without EMP (7 vs. 12 months, respectively; $\mathrm{P}=0.555$; Fig. 2E). From Fig. 2F, it may be observed that the patients who received chemotherapy combined with bortezomib and autologous SCT had a longer median OS, the patients who received only chemotherapy without bortezomib had a shorter median OS and other patients had an intermediate OS. However, there was no statistical difference among these differently treated patients $(\mathrm{P}=0.053)$.

The median DOR was 5 months (range, 0-28 months) (Fig. 3A). The patients who received bortezomib or autologous SCT had a significantly longer median DOR than those who 
A

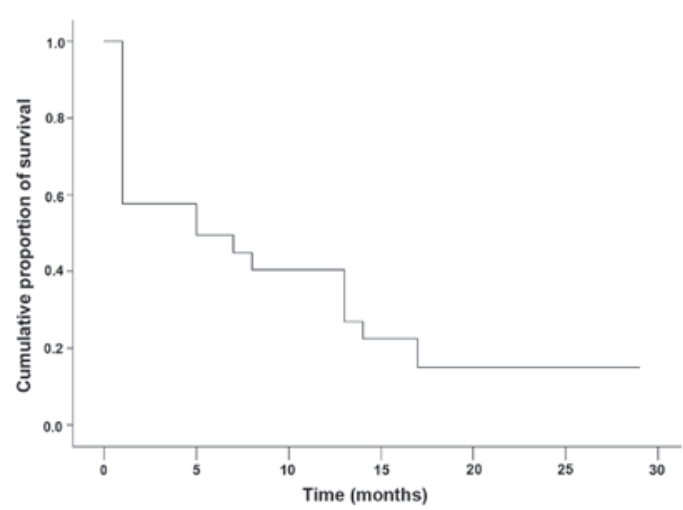

C

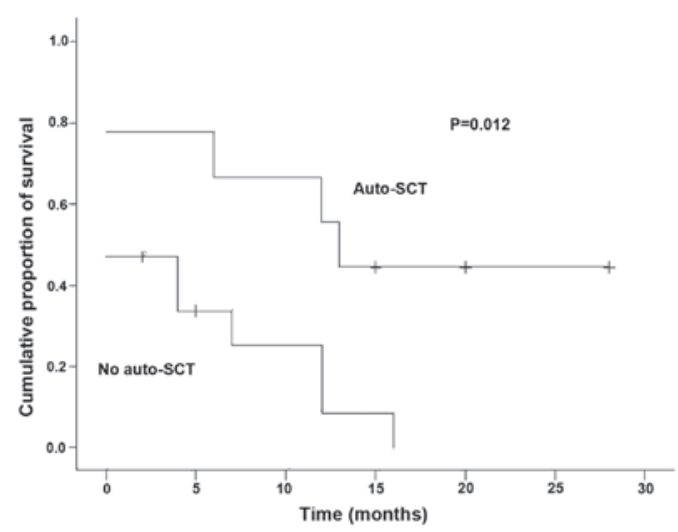

B

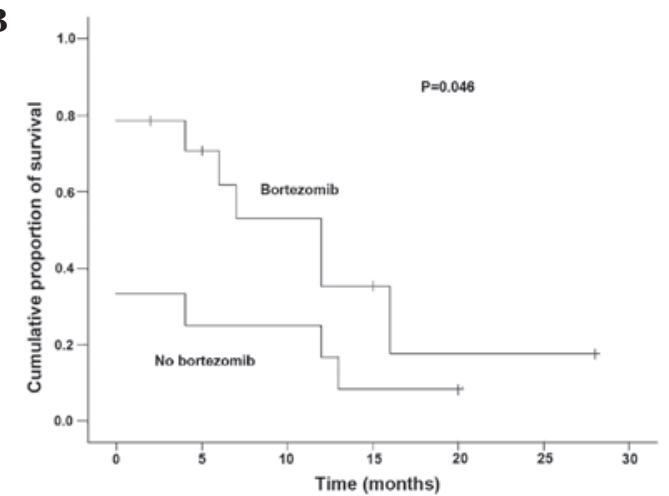

D

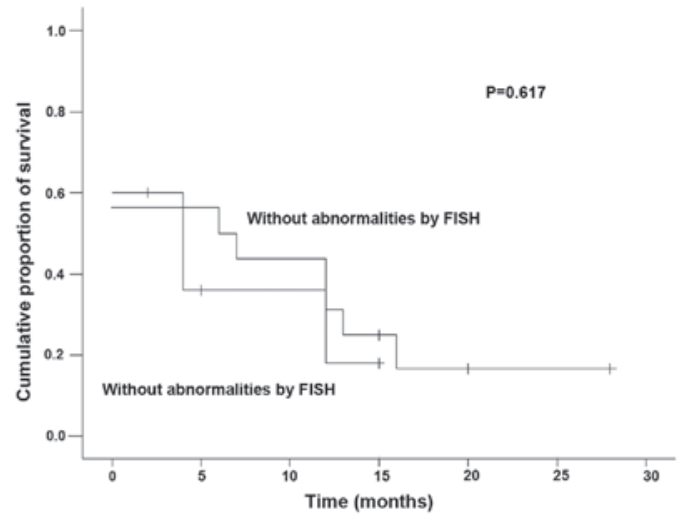

$\mathbf{E}$

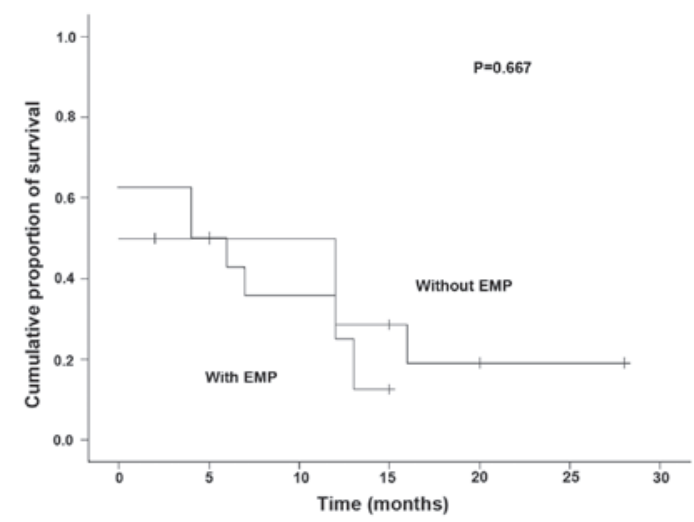

Figure 3. Duration of remission of (A) the patients with two bands for the $\lambda$ light chain on IFE (n=26) and according to the factors (B) bortezomib, (C) autologous SCT, (D) abnormalities by FISH and (E) EMP. IFE, immunofixation electrophoresis; SCT, stem cell transplantation; FISH, fluorescence in situ hybridization; EMP, extramedullary plasmacytoma.

$\operatorname{did} \operatorname{not}(\mathrm{P}=0.046$ and $\mathrm{P}=0.012$, respectively; Table III, Fig. 3B and C). However, the patients with abnormalities by FISH had a median DOR similar to that of the patients without abnormalities by FISH, and there was no statistically significant difference in median DOR between the patients with EMP and the patients without EMP $(\mathrm{P}=0.617$ and $\mathrm{P}=0.667$, respectively; Table III, Fig. 3D and E).

\section{Discussion}

In the present study, a series of patients with MM who were revealed to have rare double M-components by IFE were investigated. The two $\lambda$ light chains, as shown in Fig. 1, had different velocities during IFE; therefore, they should rationally have different molecular weights. It may be concluded that the two chains are different. It is noteworthy that the light chains of all the patients are $\lambda$ light chains. Such patients had a very poor prognosis with a median OS only 12.6 months and, therefore, it is recommended that these patients should receive particular attention.

Renal dysfunction is a common symptom that negatively affects the quality of life of the patient and their prognosis. With the clinical application of novel strategies and agents such as autologous SCT and bortezomib, this situation has improved (8). In the present study, 17 patients (65.4\%) had renal dysfunction and 12 patients $(46.2 \%)$ in the group received hemodialysis. The rates of renal dysfunction and severe renal dysfunction were higher than those reported in previous studies $(3,8)$. The pathogenesis is unknown. The pathophysiology of the most common types of renal injury observed in myeloma is closely linked to the renal handling of light chains (9). It may be concluded that the renal function of the patients is likely to be affected severely by the presence of two different $\lambda$ light chains. 
EMP is not a rare manifestation of MM and its incidence is $9-13 \%(5,10)$. Notably, $\operatorname{IgD}$ MM is associated with a higher frequency of EMP. In a study by Bladé et al, EMP was reported in $15-20 \%$ of patients at diagnosis and in an additional $15 \%$ of patients during follow-up (11). Another study described a series of 72 patients with MM aged $<40$ years with a high incidence of EMP (12). A high incidence of EMP may be associated with younger age or IgD MM. In the present study, the median age of all patients was 59 years old and IgD MM accounted for $19.2 \%(5 / 26)$ of cases. However, the incidence of EMP at diagnosis was $38.5 \%$ (10/26). This observation may explain the interesting phenomenon that $\mathrm{MM}$ in patients with two different $\lambda$ light chains appears to have a stronger extramedullary invasive ability.

The patients in the present study had a poor prognosis with a median OS of 12.6 months. Renal dysfunction, EMP and abnormalities by FISH are all known to be poor prognostic factor $(3,5,8,13)$. However, neither EMP nor the abnormalities identified by FISH were observed to affect the prognosis in the present study. The identification of two different $\lambda$ light chains by IFE at diagnosis could be an independent poor prognostic factor. To the best of our knowledge, no relevant studies concerning the therapy of such patients have been reported previously. In the present study, bortezomib and autologous SCT were found to be superior to conventional chemotherapy in the treatment of these patients.

In conclusion, patients with MM and two different $\lambda$ light chains as identified by IFE may be a special group that is affected by a high incidence of renal dysfunction and EMP. They have a poor prognosis, and novel therapies including bortezomib and autologous SCT may increase the chances of survival of such patients.

\section{References}

1. Rajkumar SV: Treatment of multiple myeloma. Nat Rev Clin Oncol 8: 479-491, 2011.

2. Greipp PR, San Miguel J, Durie BG, Crowley JJ, Barlogie B, Bladé J, et al: International staging system for multiple myeloma. J Clin Oncol 23: 3412-3420, 2005.

3. Morabito F, Gentile M, Ciolli S, et al: Safety and efficacy of bortezomib-based regimens for multiple myeloma patients with renal impairment: a retrospective study of Italian Myeloma Network GIMEMA. Eur J Haematol 84: 223-228, 2010.

4. Liu N, Zhou H, Yang G, Geng C, Jian Y, Guo H and Chen W: Retrospective analysis of genetic abnormalities and survival in 131 patients with multiple myeloma. Oncol Lett 9: 930-936, 2015.

5. Varettoni M, Corso A,Pica G, et al: Incidence, presenting features and outcome of extramedullary disease in multiple myeloma: a longitudinal study on 1003 consecutive patients. Ann Oncol 21: 325-330, 2010.

6. Fonseca R, Monge J and Dimopoulos MA: Staging and prognostication of multiple myeloma. Expert Rev Hematol 7: 21-31, 2014.

7. Durie BG, Harousseau JL, Miguel JS, et al; International Myeloma Working Group: International uniform response criteria for multiple myeloma. Leukemia 20: 1467-1473, 2006.

8. Yang GZ and Chen WM: Bortezomib-based regimen for the treatment of 110 multiple myeloma patients. Zhonghua Yi Xue Za Zhi 90: 2671-2674, 2010 (In Chinese).

9. Dimopoulos MA, Kastritis E, Rosinol L, Bladé J and Ludwig H: Pathogenesis and treatment of renal failure in multiple myeloma. Leukemia 22: 1485-1493, 2008.

10. Chen HF, Wu TQ, Li ZY, et al: Extramedullary plasmacytoma in the presence of multiple myeloma: clinical correlates and prognostic relevance. Onco Targets Ther 5: 329-334, 2012.

11. Bladé J, Lust JA and Kyle RA: Immunoglobulin D multiple myeloma: presenting features, response to therapy, and survival in a series of 53 cases. J Clin Oncol 12: 2398-2404, 1994.

12. Bladé J, Kyle RA and Greipp P: Presenting features and prognosis in 72 patients with multiple myeloma who were younger than 40 years. Br J Haematol 93: 345-351, 1996.

13. Dimopoulos M, Kyle R, Fermand JP, et al; International Myeloma Workshop Consensus Panel 3: Consensus recommendations for standard investigative workup: report of the International Myeloma Workshop Consensus Panel 3. Blood 117: 4701-4705, 2011. 